\title{
Activation of Superficial Dorsal Horn Neurons in the Mouse by a PAR-2 Agonist and 5-HT: Potential Role in Itch
}

\author{
Tasuku Akiyama, Austin W. Merrill, Mirela Iodi Carstens, and E. Carstens \\ Department of Neurobiology, Physiology, and Behavior, University of California, Davis, Davis, California 95616
}

Itch, an unpleasant sensation associated with the desire to scratch, is symptomatic of dermatologic and systemic disorders that often resist antihistamine treatment. Histamine-independent itch mediators include serotonin (5-HT) and agonists of the protease-activated receptor-2 (PAR-2). We used behavior, Fos immunohistochemistry, and electrophysiology to investigate if these mediators activate spinal dorsal horn neurons in a manner consistent with itch. Intradermal (id) injection of the PAR-2 agonist SLIGRL-NH ${ }_{2}$ in the rostral back evoked bouts of directed hindlimb scratches over 20 -30 min. Hindpaw injection of SLIGRL- $\mathrm{NH}_{2}$ produced Fos staining in superficial dorsal horn which was then targeted for single-unit recording. Small id microinjections of SLIGRL-NH ${ }_{2}$ or 5-HT identified responsive single units in the superficial dorsal horn of mice anesthetized with pentobarbital. Thirty-eight units characterized as wide dynamic range, nociceptive specific, or mechanically insensitive exhibited significantly increased firing after id SLIGRL- $\mathrm{NH}_{2}$ for 9 min, to partial (25\%) tachyphylaxis with repeated injection. A majority additionally responded to 5-HT (70\%), mustard oil (79\%), and capsaicin (71\%). Seven units isolated with the 5-HT search stimulus exhibited significant and prolonged responses to 5-HT with tachyphylaxis to repeated injections. The majority also responded to SLIGRL- $\mathrm{NH}_{2}$, mustard oil, and capsaicin. The prolonged responses of superficial dorsal horn neurons to SLIGRL-NH $\mathrm{N}_{2}$ and 5-HT suggest a role in signaling itch. However, their responsiveness to algogens is inconsistent with itch specificity. Alternatively, such neurons may signal itch, whereas noxious stimulus levels recruit these and a larger population of pruritogen-insensitive cells to signal pain which masks or occludes the itch signal.

\section{Introduction}

Chronic itch is a common clinical problem associated with a variety of dermatologic conditions such as atopic dermatitis, as well as kidney and liver disease (Ikoma et al., 2006). Itch is often poorly controlled by antihistamines (Twycross et al., 2003), and there is a pressing need for improved treatments of chronic itch. The protease-activated receptor-2 (PAR-2) has been implicated in pain and inflammation (Cottrell et al., 2003; Dai et al., 2004), as well as itch (Steinhoff et al., 2003). PAR-2 agonists elicit doserelated scratching in mice (Shimada et al., 2006; Ui et al., 2006; Iodi Carstens et al., 2008) consistent with a role for PAR-2 in peripheral itch transduction. PAR-2 may represent a nonhistaminergic mechanism of itch, offering a novel target for development of antipruritic treatments. The bean plant, cowhage, has pods with spicules containing a protease, mucunain, that induces itch without flare when applied to human skin (Johanek et al., 2007) and excites C-polymodal nociceptors (Namer et al., 2008). Mucunain was recently shown to act at PAR-2 and PAR-4 (Reddy et al., 2008). In contrast, histamine, the prototypical itch mediator in human skin, induces a local flare and itch sensation via activation of mechanically insensitive C-fibers (Schmelz et al., 1997), which in turn contact histamine-responsive spinothalamic tract (STT) neurons in the superficial spinal dorsal horn

\footnotetext{
Received Dec. 22, 2008; revised Feb. 9, 2009; accepted March 4, 2009.

This work was supported by National Institutes of Health Grant DE013685 and AR057194.

Correspondence should be addressed to Prof. E. Carstens, Department of Neurobiology, Physiology, and Behav-

ior, University of California, Davis, 1 Shields Avenue, Davis, CA 95616. E-mail: eecarstens@ucdavis.edu.

DOI:10.1523/JNEUROSCI.6103-08.2009

Copyright $\odot 2009$ Society for Neuroscience $\quad$ 0270-6474/09/296691-09\$15.00/0
}

(Andrew and Craig, 2001) that transmit itch signals to higher centers. Furthermore, itch evoked by histamine and cowhage may activate separate subpopulations of primary afferent C-fibers (Namer et al., 2008) and STT neurons (Davidson et al., 2007). Since most behavioral studies of itch mechanisms use mice, we investigated if a PAR-2 agonist activates neurons in superficial laminae of the murine spinal cord dorsal horn in a manner consistent with a role in signaling itch.

Serotonin (5-hydroxytryptamine, 5-HT) is another inflammatory mediator that elicits dose-dependent scratching behavior in rats (Thomsen et al., 2001; Jinks and Carstens, 2002; Nojima and Carstens, 2003a,b; Nojima et al., 2003) and mice (Yamaguchi et al., 1999; Cuellar et al., 2003). Intraplantar injection of 5-HT elicits dose-related and naltrexone-sensitive biting and licking directed to the injection site but not limb scratching (Hagiwara et al., 1999). This suggests that itch in distal extremities is associated with biting, gnawing, or licking, which may substitute for scratching to relieve itch and justifies investigating itch mechanisms in lumbar neurons receiving hindpaw afferents. 5-HT excites rat superficial dorsal horn neurons over a prolonged time course matching scratching behavior, although most units also responded to algogenic stimuli (Jinks and Carstens, 2002). We presently also investigated if 5-HT excites mouse superficial dorsal horn neurons in a manner consistent with itch. We used a chemical search strategy (Jinks and Carstens, 2000, 2002) to increase the likelihood of identifying neurons responsive to the PAR-2 agonist or 5-HT. We also tested their chemical selectivity by determining if they additionally responded to mustard oil and capsaicin, which elicit sensations of burning pain rather than itch. 


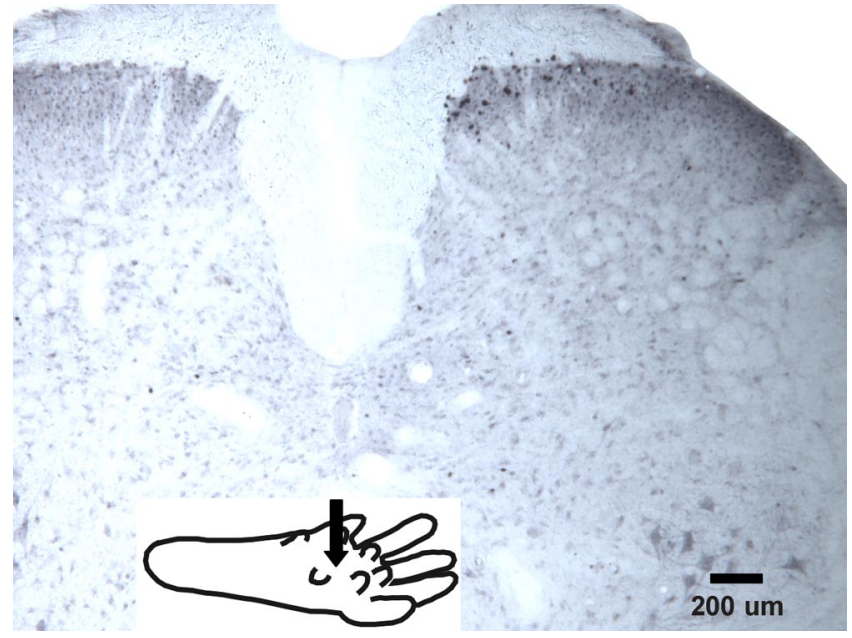

Figure 1. FLI in lumbar superficial dorsal horn after intraplantar microinjection of PAR-2 agonist SLIGRL-NH 2 . Photomicrograph of lumbar section showing FLI (black nuclei) after intraplantar microinjection of PAR-2 agonist SLIGRL-NH ${ }_{2}(50 \mu \mathrm{g}$ in $5 \mu \mathrm{l})$. Arrow in inset shows injection site on ipsilateral hindpaw.

An abstract of parts of this study has appeared previously (Carstens et al., 2008).

\section{Materials and Methods}

All experiments were conducted using adult male ICR mice (Harlan) (25-52 g) under a protocol approved by the University of California, Davis Animal Care and Use Committee.

Fos expression. Mice were anesthetized with sodium pentobarbital (60 $\mathrm{mg} / \mathrm{kg}$, i.p.). A unilateral intraplantar microinjection of SLIGRL-NH (50 $\mu \mathrm{g}$ in $5 \mu \mathrm{l}$ of isotonic saline; Bachem) or vehicle (isotonic saline) was made, and the animal was perfused transcardially $2 \mathrm{~h}$ later with PBS followed by $4 \%$ paraformaldehyde as described previously (Merrill et al., 2006). The lumbosacral spinal cord was removed and postfixed for $8 \mathrm{~h}$, cryoprotected in a $30 \%$ sucrose solution, and cut in $50 \mu \mathrm{m}$ sections. Every third section was washed and blocked in goat serum (3\%) and then incubated in primary c-Fos antibody (1:50,000; Arnel Products) for $2 \mathrm{~d}$. Sections were then treated with a secondary biotinylated (goat-antirabbit) antibody followed by an avidin-biotin-peroxidase complex reaction enhanced with biotinyl tyramide $/ \mathrm{H}_{2} \mathrm{O}_{2}$. Immunoreactivity was visualized using a nickel-enhanced diaminobenzidine reaction. Sections were mounted on slides, coverslipped, and examined by a blinded investigator under the light microscope (Nikon E-200). Counts of cells in the superficial dorsal horn expressing Fos-like immunoreactivity (FLI) were averaged for five sections per animal and between-group comparisons made by ANOVA.

Scratching behavior. Animals were habituated to the Plexiglas recording arena in three daily $1 \mathrm{~h}$ sessions. They then received an intradermal (id) injection of SLIGRL- $\mathrm{NH}_{2}$ (50 $\mu \mathrm{g} / 5 \mu \mathrm{l}$ in isotonic saline) or vehicle (isotonic saline) in the rostral back and immediately placed in the arena and videotaped for $45 \mathrm{~min}$, as described in a previous study (Cuellar et al., 2003). Tests using the same mouse were conducted at least 1 week apart. Bouts of hindlimb scratching directed toward the injection site were counted in $5 \mathrm{~min}$ intervals. Spontaneous scratching was similarly counted in the same animals (no treatment). We used number of scratch bouts, rather than other parameters such as within-bout scratching frequency, because only counts of scratch bouts were significantly correlated with the concentration of the pruritic stimulus (Nojima and Carstens, 2003b). In contrast, the within-bout frequency of individual scratches $(\sim 12 \mathrm{~Hz}$ in mice), and duration of scratch bouts $(\sim 1.5-3 \mathrm{~s})$, remained constant across various stimulus conditions and did not correlate significantly with stimulus intensity (Nojima and Carstens, 2003b).

Electrophysiology. Anesthesia was induced with sodium pentobarbital $(60 \mathrm{mg} / \mathrm{kg}$, i.p.) and maintained by constant infusion of pentobarbital through a jugular cannula at a rate sufficient to maintain areflexia. The
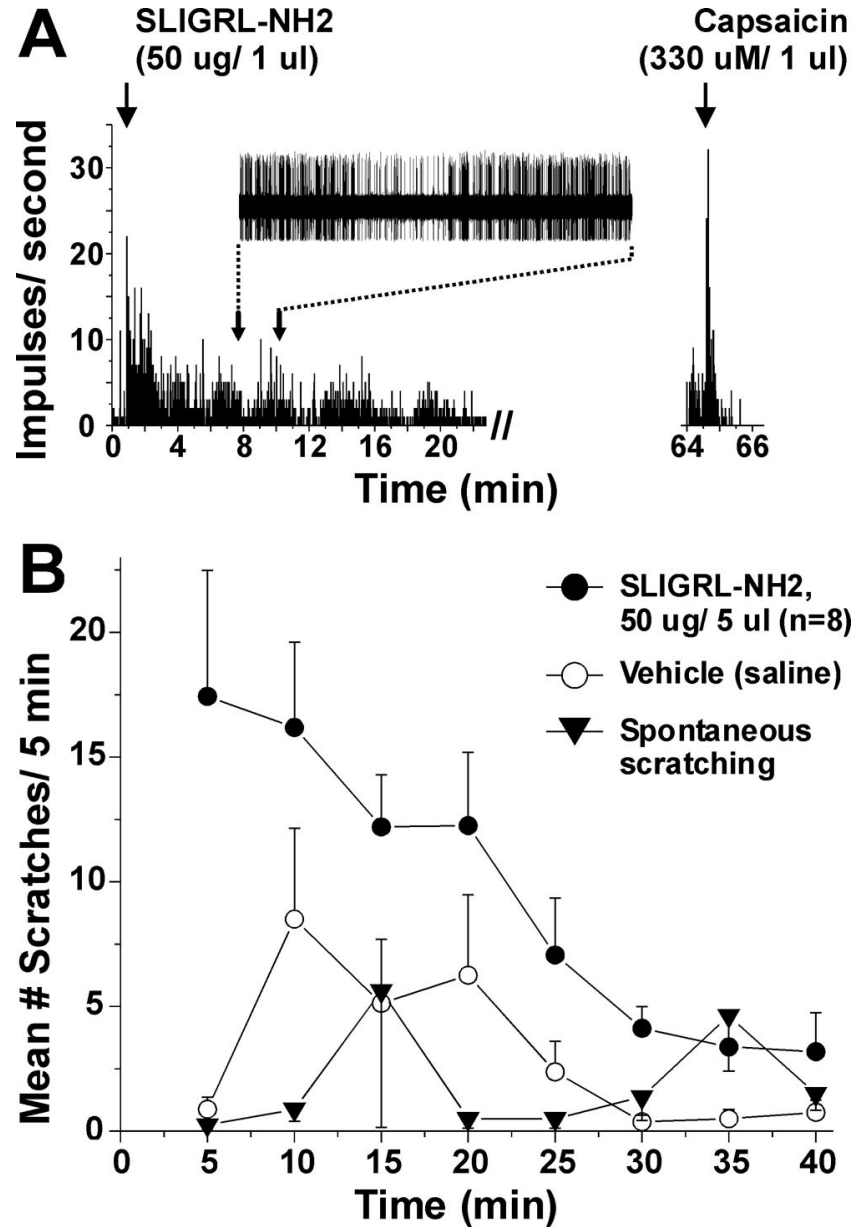

Figure 2. Excitation of superficial lumbar dorsal horn unit by SLIGRL-NH ${ }_{2}$ over a time course consistent with scratching. $A$, PSTH (1 s bins) of superficial lumbar dorsal horn unit's response to intraplantar id microinjection of SLIGRL-NH 2 (arrow). PSTH (right) shows same unit's response to id capsaicin. Inset shows train of action potentials. Time axis is aligned with graph of scratching shown in $\boldsymbol{B}$. Note that unit firing increased after injection and persisted for $20 \mathrm{~min}$. $\boldsymbol{B}$, Graph plots mean number of scratch bouts versus time. : Mice received id microinjection of SLIGRL$\mathrm{NH}_{2}$ into the nape of the neck at time 0 . Bouts of hindlimb scratching directed toward the injection site were counted at 5 min intervals. Scratching peaked at $5 \mathrm{~min}$ after injection and persisted for $25 \mathrm{~min}$. Error bars indicate SEM; $n=8$. $\bigcirc$ : Vehicle (isotonic saline; tested separately in same mice). $\mathbf{\nabla}$ : Spontaneous scratching in same group of mice. There was a significant difference in mean number of scratch bouts over time between SLIGRL-NH 2 -evoked and spontaneous scratching $(p<0.05$, ANOVA)

lumbosacral spinal cord was exposed by laminectomy and the animal fixed in a stereotaxic frame as described previously (Cuellar et al., 2004). A tungsten microelectrode (FHC) was driven into the spinal cord by hydraulic microdrive (David Kopf Instruments) to record extracellular single-unit activity which was amplified, digitized (Powerlab; AD Instruments), and displayed on-line using Chart 5 software (AD Instruments).

A chemical search strategy (Jinks and Carstens, 2000, 2002) was used to isolate units in the superficial dorsal horn. A 30-gauge needle connected to PE 10 tubing filled with either SLIGRL-NH $2(50 \mu \mathrm{g} / \mu \mathrm{l}$ in saline) or $5-\mathrm{HT}(14 \mathrm{~mm} / \mu$ lin saline) was inserted id in the plantar skin. A small volume $(\sim 0.25 \mu \mathrm{l})$ was microinjected, and the spinal recording electrode was advanced until a unit exhibiting ongoing firing was isolated. If no unit was isolated, the procedure was repeated at least $10 \mathrm{~min}$ later at a different site on the plantar surface, or on the opposite side. When an active unit was isolated, we then waited until firing decreased to zero or a steady low level over a period of at least $10 \mathrm{~min}$, based on the assumptions that (1) the unit had a low level of activity before the search stimulus, (2) ongoing activity was attributable to injection of the search stimulus, and (3) stimulus-evoked activity would eventually decline to 
Table 1. Incidence of activation of SLIGRL-NH $\mathrm{NH}_{2}$-responsive units by other stimuli tested

\begin{tabular}{llllll}
\hline Brush & Pinch & Heat & Cold & 5-HT & Capsaicin \\
\hline $68 \%(26$ of 38$)$ & $95 \%(36 \text { of } 38)^{a}$ & $78 \%(28$ of 36$)$ & $53 \%(19$ of 36$)$ & $70 \%(14$ of 20$)$ & $79 \%(23$ of 29$)$ \\
\hline
\end{tabular}

Sixty-eight percent were classified as WDR and $27 \%$ as NS. Heat stimulus was $44-54^{\circ} \mathrm{C}$ over 30 s, cold was $36-0^{\circ} \mathrm{C}$ over 30 s, by Peltier thermode. Mustard oil (70\%) applied topically, 5 -HT and capsaicin injected intradermally. Responsive units exhibited $>30 \%$ increase in spikes per minute after stimulus versus prestimulus baseline.

${ }^{a}$ Two units unresponsive to pinch were unresponsive to brush with cotton $=$ mechano-insensitive.
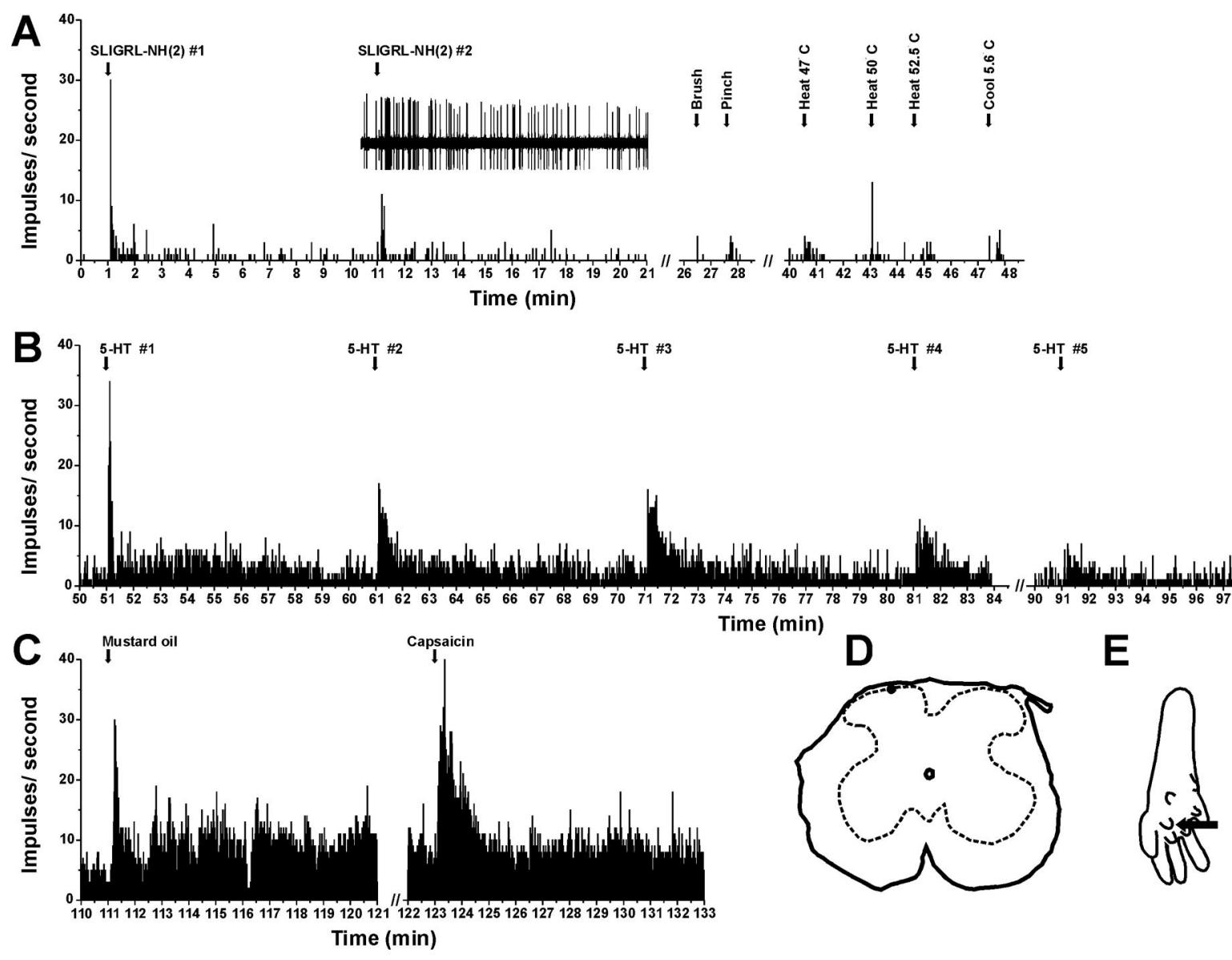

Figure 3. Example of unit identified by SLIGRL-NH 2 search stimulus. A, PSTH shows successive responses to id SLIGRL-NH 2 (arrows), followed by mechanical and thermal stimuli. Inset, Raw action potentials. $\boldsymbol{B}$, Continued recording from unit in $\boldsymbol{A}$ shows responses to successive id 5 -HT and lack of response to vehicle (saline). $\boldsymbol{C}$, Responses to mustard oil, capsaicin. $\boldsymbol{D}$, Recording site in lamina I. $\boldsymbol{E}$, Arrow shows injection site.

close to the presearch level. SLIGRL- $\mathrm{NH}_{2}$ or 5 -HT $(1 \mu \mathrm{l})$ was then reinjected. The $1 \mu \mathrm{l}$ injection volume was chosen consistent with our previous studies (Carstens, 1997; Jinks and Carstens, 2000, 2002), allowing repeated injections at the same site on the assumption that the small volume is rapidly cleared from the id injection site. Units exhibiting increased firing ( $>30 \%$ above preinjection baseline) were studied further. In most cases, the same chemical (i.e., either SLIGRL-NH $\mathrm{N}_{2}$ or 5-HT) was reinjected twice more at $10 \mathrm{~min}$ interstimulus intervals to test for tachyphylaxis. Then, the injection needle was removed and replaced with another one for microinjection of a different chemical. In most $(n=54)$ experiments, units were searched with SLIGRL- $\mathrm{NH}_{2}$ and tested usually with three (but occasionally 2, 4, or 5) successive injections, followed by three successive microinjections of 5-HT. In some experiments $(n=17)$, 5 -HT was used as a search stimulus, and the same stimulus sequence was followed in reverse order.

After completion of testing with SLIGRL- $\mathrm{NH}_{2}$ and 5-HT, unit receptive fields were mapped using mechanical (touch-pressure) stimuli. Since we used a chemical search strategy, it was not possible to assess mechanical or thermal sensitivity before the pruritogen search stimulus was injected. This approach also avoided any potential effects of the physical stimuli on pruritogen-evoked neuronal activity. Units were classified as wide dynamic range (WDR) if they responded to heat and responded at higher firing rate to pinch than light touch. They were classified as nociceptive specific (NS) if they responded to pinch (and often also noxious heat) but not light touch. Two units were mechanically insensitive. An array of additional mechanical, thermal, and chemical stimuli was then delivered. Mechanical stimuli included light brushing with a cotton wisp, pinch with forceps, and graded mechanical indentation with a series of calibrated von Frey filaments (usually 4, 12, and $76 \mathrm{~g}$ ). Thermal stimuli included brief $(10 \mathrm{~s})$ increases in temperature up to $44-54^{\circ} \mathrm{C}$, or cooling down to $0^{\circ} \mathrm{C}$, from an adapting temperature of $34^{\circ} \mathrm{C}$, delivered by a computer-controlled Peltier thermode (Physitemp NTE-2A). Chemical stimuli included isotonic saline ( $1 \mu \mathrm{l}$ id; vehicle control), mustard oil ( $75 \%$ in mineral oil, delivered topically in a $2 \mu$ l volume), capsaicin (3.3 $\mathrm{mm} / 1 \mu \mathrm{l})$, and in a few experiments, histamine $(1 \% / 1 \mu \mathrm{l})$. Mechanical and thermal stimuli were always tested before other chemical stimuli; mustard oil was usually applied before capsaicin, but in a few experiments, the order was reversed.

Unit action potentials were counted using Chart software. Unit activity was usually quantified as number of action potentials per minute and displayed in peristimulus-time histogram (PSTH) format with $1 \mathrm{~s}$ bins. Group responses at $1 \mathrm{~min}$ intervals after a given stimulus were compared 
with activity 1 min before the stimulus by repeated-measures ANOVA (PSS 9.0), with $p<$ 0.05 set as significant.

At the conclusion of recording, an electrolytic lesion was made. The spinal cord was fixed in $10 \%$ buffered formalin, and $50 \mu \mathrm{m}$ sections were cut and mounted on slides for microscopic verification of the lesion site.

\section{Results}

\section{Spinal Fos expression}

We initially used Fos expression to identify locations of lumbar neurons activated by intraplantar microinjection of the PAR-2 agonist SLIGRL-NH $\mathrm{N}_{2}$. Figure 1 shows a typical example of FLI located superficially in laminae I-II. No FLI was observed in the contralateral dorsal horn. Counts of FLI in the ipsilateral superficial dorsal horn after SLIGRL- $\mathrm{NH}_{2}$ were significantly greater compared with vehicle (saline)-treated controls [PAR-2 agonist: mean, 15.28 per section \pm 2.25 (SEM); saline: 1.1 per section \pm 0.97 , respectively; $p<0.05$ ], the low number of which was similar to that observed in our previous study (Merrill et al., 2006).

\section{Scratching behavior}

We and others (Shimada et al., 2006; Ui et al., 2006; Iodi Carstens et al., 2008) have shown that the PAR-2 agonist SLIGRL$\mathrm{NH}_{2}$ elicits dose-related scratching. Figure 2 shows comparable time courses of neuronal (Fig. 2A) and behavioral (Fig. 2B) responses to id injection of SLIGRL- $\mathrm{NH}_{2}$ over a $20 \mathrm{~min}$ period. The superficial dorsal horn unit shown in Figure $2 A$ additionally responded to capsaicin (right, PSTH). Figure $2 B$ shows the time course of scratch bouts elicited by id injection of SLIGRL$\mathrm{NH}_{2}(50 \mu \mathrm{g})$ into the nape of the neck, along with vehicle-evoked and spontaneous scratching. The total number of SLIGRL-NH $\mathrm{N}_{2}$ evoked scratch bouts was significantly greater compared with vehicle or untreated (spontaneous scratching) conditions $(p<$ $0.01 ; p<0.05$, respectively).

\section{Electrophysiology: PAR-2 agonist search \\ Unit characterization}

We then targeted the superficial dorsal horn using a chemical search strategy to identify units responsive to SLIGRL- $\mathrm{NH}_{2}$. Thirty-eight of fifty-four (70\%) units responded to a second id injection of SLIGRL- $\mathrm{NH}_{2}$ given more than 10 min later. Of these, $68 \%$ were classified as WDR type, $27 \%$ as NS, and 5\% were mechanically insensitive (Table 1). The majority of units additionally responded to noxious heating, cooling, and other irritant chemical stimuli (Table 1). It should be cautioned, however, that mechanical and thermal responses might have been affected by previous application of the PAR-2 agonist, which was unavoidable using our search strategy. A typical example of a unit responsive to all irritant chemical stimuli is shown in Figure 3. Most unit recording sites were in the superficial dorsal horn based on micrometer depth (mean, 189.1 $\mu \mathrm{m} \pm 24.8$ SEM), and histologically identified recordings sites were mainly in lamina I (Fig. $4 B$, inset).
SLIGRL- $\mathrm{NH}_{2}$

Responses to SLIGRL- $\mathrm{NH}_{2}$ usually consisted of an initial highfrequency component adapting to a steady-state level that sometimes persisted for $10 \mathrm{~min}$ or more (Figs. $2 \mathrm{~A}, 3 \mathrm{~A}$ ). Figure $4 \mathrm{~A}$ shows averaged responses of 18 units to three repeated injections of SLIGRL-NH $\mathrm{N}_{2}$. After the first injection, the mean firing rate increased significantly relative to preinjection baseline $\left(F_{(1,17)}=\right.$ 58.8, $p<0.001$ ) out to $9 \mathrm{~min}$ after injection (Fig. $4 A$ ). After the second injection of SLIGRL- $\mathrm{NH}_{2}$, mean firing was significantly $(p<0.001)$ elevated for $5 \mathrm{~min}$ (i.e., to minute 16 in Fig. $4 A$ ) before returning to baseline. After the third injection, mean firing was also significantly $(p<0.001)$ elevated for $4 \mathrm{~min}$ (i.e., to minute 25 in Fig. $4 A$ ). It is also apparent from Figure $4 A$ that the second and third responses to SLIGRL- $\mathrm{NH}_{2}$ were lower compared with the first, indicating tachyphylaxis. This is confirmed in Figure 5, which plots individual (thin lines) and mean (thick line) unit responses to three successive injections of SLIGRL$\mathrm{NH}_{2}$ at 10 min intervals. Most (but not all) units exhibited a lower response to the second versus first injection, and on average, the second response was significantly lower (by $24.8 \%$ ) than the first $(p<0.05$, paired $t$ test) but did not differ significantly from the third response (Fig. 5).

\section{5-HT}

Seventy percent of the PAR-2 agonist-responsive units tested also responded to id 5-HT (Table 1 ). Figure $3 B$ shows an individual unit's responses to five repeated injections of 5-HT at $10 \mathrm{~min}$ 


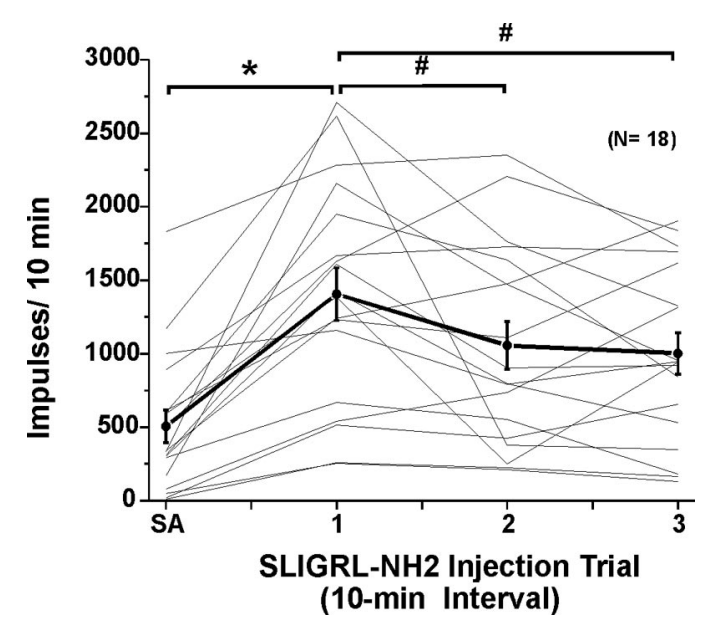

Figure 5. Responses of lumbar superficial dorsal horn units to repeated id microinjections of SLIGRL-NH 2 . Thin lines plot each individual unit's SA (recorded for $1 \mathrm{~min} \times 10$ ) and response to three repeated id microinjections of SLIGRL-NH 2 at 10 min intervals. Thick black line connecting dots with error bars (SEM) shows mean of 18 units tested. *Mean response to first injection of SLIGRL-NH ${ }_{2}$ significantly different from SA ( $p<0.05$, ANOVA). "Second and third responses to SLIGRL-NH ${ }_{2}$ significantly different from first ( $p<0.05$ ); i.e., tachyphylaxis. Third mean response not significantly different from second; second and third responses significantly different from $S A$.

intervals. Responses were characterized by an initial highfrequency discharge followed by a prolonged period of elevated firing, with gradual tachyphylaxis to repeated injections. Figure $4 B$ plots mean responses of six units to repeated 5-HT. After each 5 -HT injection, the mean firing per minute was significantly $(p<0.5)$ elevated for the first minute. Successive responses to 5-HT decreased, indicating tachyphylaxis. When averaged over the 10 min postinjection period, the second 5-HT response was significantly lower than the first $(p<0.001)$, and the third was significantly lower than the second $(p<0.001)$.

\section{Other stimuli}

The majority of units additionally responded to the other mechanical, thermal, and chemical stimuli tested (Table 1). Figure $4 C$ shows mean responses of SLIGRL- $\mathrm{NH}_{2}$-responsive units to additional stimuli. Both mustard oil and capsaicin elicited significant increases in firing ( $p<0.01$ and $p<0.05$, respectively) during the first minute after injection compared with preinjection baseline. Similarly, brush and pinch stimuli both significantly increase firing averaged over $10 \mathrm{~s}$, and heating and cooling significantly increased firing over $30 \mathrm{~s}$, whereas saline did not significantly affect the firing rate.

\section{5-HT search}

Seventeen units were isolated using an id 5-HT search stimulus, and seven $(41 \%)$ responded to subsequent injection of 5-HT (WDR, six; NS, one). The mean recording depth was $172.8 \mu \mathrm{m}$ \pm 36.4 (SEM), and histologically recovered sites were in lamina I (Fig. $4 B$, inset).

Figure $6 A$ shows a WDR unit that responded to the initial 5-HT with a prolonged and variable increase in firing. It responded weakly to a second injection of 5-HT followed by variable prolonged activity. This WDR unit located in lamina I (Fig. 6C) responded with phasic, decrementing responses to repeated injections of SLIGRL-NH $\mathrm{NH}_{2}$ (Fig. $6 \mathrm{~B}$ ) and additionally responded to histamine, mustard oil, and capsaicin (Fig. 6D). Figure $7 A$ shows averaged responses of seven units to three successive applications of 5-HT. After the first injection of 5-HT, mean firing per minute was significantly greater for 9 min compared with the pre-5-HT baseline ( $p<0.01$ for all time points). After the second 5-HT injection, mean firing during the first min (Fig. 7A, min $11-12)$ differed significantly $(p<0.05)$ from baseline activity preceding the initial 5-HT injection (minute $0-1$ ) but not from activity during the immediately preceding minute (minute 1011). This indicates that the increase in firing elicited by the first 5-HT injection had not completely returned to baseline before the second 5-HT injection was made. There was no significant increase in mean firing above baseline at any time after the third 5 -HT injection. Figure 8 plots individual (thin lines) and mean (thick line) responses to 5-HT. Mean activity after the first and second 5-HT injections did not differ significantly from each other, with both significantly greater $(p<0.05)$ than baseline [spontaneous activity (SA)]. Mean activity after the third 5-HT injection was significantly lower $(p<0.05)$ compared with the first and second 5-HT injections and did not differ significantly from baseline (SA).

Most (80\%) of the 5-HT-responsive units also responded to subsequent SLIGRL-NH $\mathrm{N}_{2}$ (Fig. $6 B$ ). Figure $7 B$ shows mean responses to three successive injections of SLIGRL-NH $\mathrm{N}_{2}$. Mean firing per minute significantly increased for $2 \mathrm{~min}$ after the first and second injections $(p<0.01)$ and increased for $1 \mathrm{~min}$ after the third injection $(p<0.01)$, after which it decreased $(p<0.05)$. When averaged over the $10 \mathrm{~min}$ postinjection period, mean responses to the first and second injections of SLIGRL- $\mathrm{NH}_{2}$ were not significantly different, whereas the third response was significantly lower compared with the second $(p<0.001)$.

Averaged responses to the additional stimuli tested are shown in Figure 7C. All 5-HT-responsive units tested also responded to mustard oil, capsaicin, and noxious heat, and $80 \%$ responded to cooling. Evoked responses averaged over $60 \mathrm{~s}$ (mustard oil and capsaicin), $10 \mathrm{~s}$ (brush and pinch), or $30 \mathrm{~s}$ (heat and cool) were all significantly greater $(p<0.05)$ compared with prestimulus baseline. Saline injection did not have any significant effect.

\section{Discussion}

The present study identified populations of WDR and NS neurons in the superficial dorsal horn that exhibited prolonged responses to the PAR-2 agonist SLIGRL- $\mathrm{NH}_{2}$, as well as 5-HT, suggesting a possible role in the transmission of itch. However, the vast majority of these units additionally responded to noxious stimuli including mustard oil and/or capsaicin that are generally associated with burning pain rather than itch. These data are discussed in terms of the potential role of such neurons to signal itch versus pain.

\section{PAR-2}

PAR-2 has been implicated in pain and inflammation (Cottrell et al., 2003; Dai et al., 2004), as well as itch (Steinhoff et al., 2003). PAR-2 agonists elicit dose-related scratching in mice (Shimada et al., 2006; Ui et al., 2006; Iodi Carstens et al., 2008), consistent with a role in itch rather than pain, since scratching would tend to enhance pain and thus be avoided. The present data confirm recent studies showing that the PAR-2 agonist SLIGRL- $\mathrm{NH}_{2}$ induces Fos expression in murine superficial dorsal horn neurons (Dai et al., 2004; Nakano et al., 2008) and extend them by showing electrophysiologically that a subpopulation of superficial neurons is excited by this agonist. Neuronal responses continued for $9 \mathrm{~min}$ on average and often longer. This contrasts with the shorter duration of capsaicin-evoked responses. However, mustard oil also elicited prolonged neuronal firing (Figs. $3 C, 4 C, 6 D$, $7 C)$, mitigating against the argument that itch is signaled by 


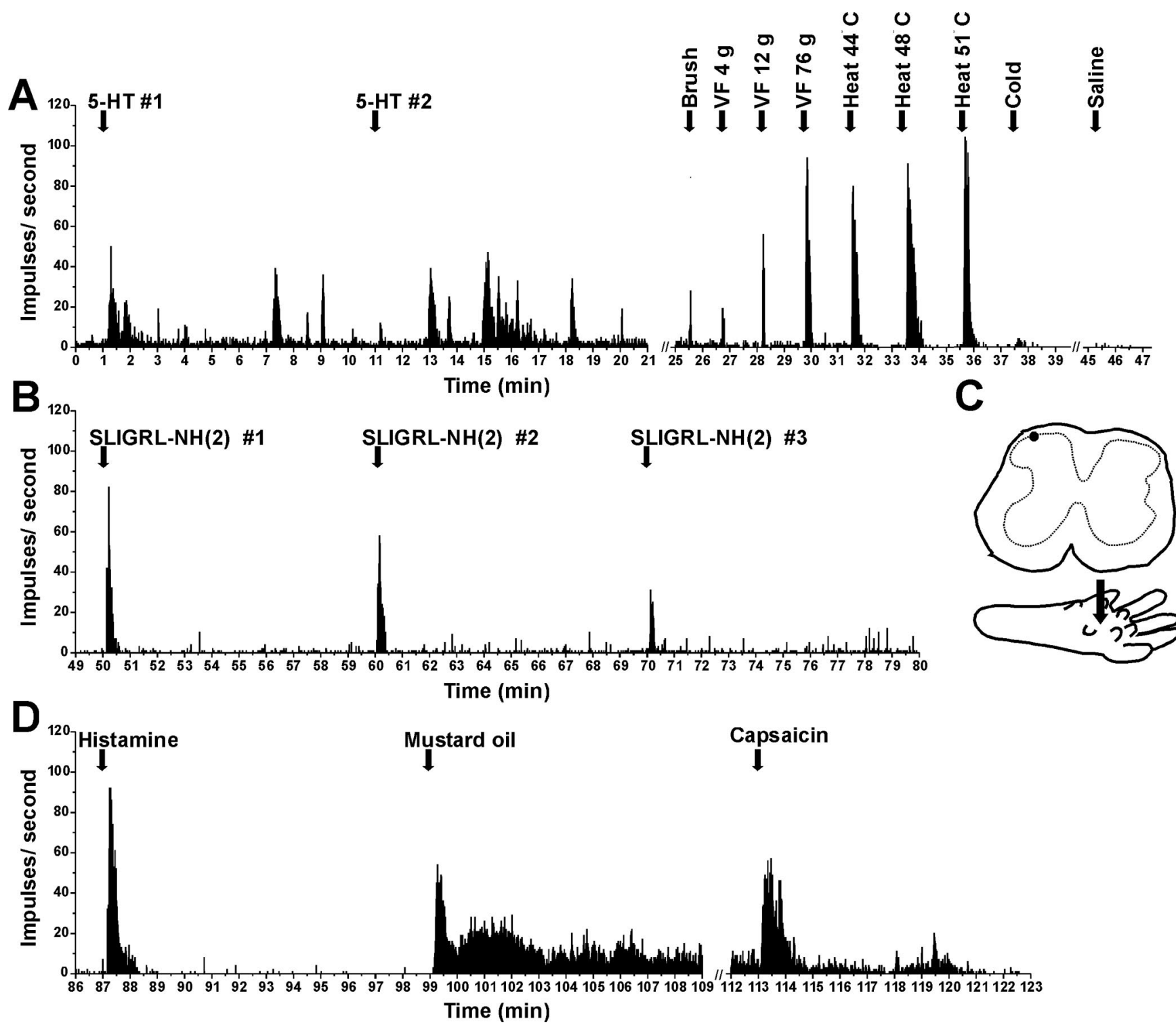

Figure 6. Individual example of responses of lamina I neuron isolated by 5 -HT search stimulus (format as in Fig. 3). $\boldsymbol{A}$, Responses to $5-\mathrm{HT}$ (14 mM/1 $\mu \mathrm{ll}$ ), mechanical and thermal stimuli, and lack of response to saline. $\boldsymbol{B}$, Successive responses to SLIGRL-NH 2 (same unit as in $\boldsymbol{A}$ ). C, Lamina I recording site (top) and intraplantar microinjection site (bottom). $\boldsymbol{D}$, Responses to id histamine (1\%/1 $\mu l)$, topical mustard oil, and id capsaicin.

longer-duration (and possibly lower frequency) neuronal firing, as opposed to a shorter-duration (and possibly higher frequency)-firing pattern for pain.

Cowhage, a bean plant whose pods have spicules containing the protease mucunain, induces nonhistaminergic itch in human skin (Johanek et al., 2007). Mucunain was recently shown to act at PAR-2 and -4 (Reddy et al., 2008), and we speculate that cowhage excites peripheral sensory fibers expressing PAR-2 to, in turn, excite superficial dorsal horn neurons that signal itch from cowhage. Recent human microneurography studies indicate the existence of separate populations of histamine- and cowhagesensitive primary afferent $\mathrm{C}$-fibers. Histamine excites mechanically insensitive $\mathrm{C}$-afferents having widespread receptive fields over a time course matching concomitant itch sensation (Schmelz et al., 1997; Namer et al., 2008), whereas cowhage excites mechanically sensitive C-fiber polymodal nociceptors (Namer et al., 2008). Interestingly, most of the cowhage- and histamine-sensitive fibers were also excited by capsaicin, consistent with our data on spinal neurons. Subpopulations of identi- fied primate STT neurons responded to either histamine or cowhage but not both (Davidson et al., 2007), consistent with the human C-fiber data and nearly all histamine- or cowhagesensitive STT neurons additionally responded to capsaicin. In monkey, many C-polymodal nociceptors were excited by both histamine and cowhage, whereas relatively few were additionally activated by capsaicin (Johanek et al., 2008). We are currently investigating if histamine and the PAR-2 agonist excite separate populations of mouse superficial dorsal horn neurons. In any event, the bulk of data suggests the existence of separate neural mechanisms and pathways for itch elicited by histamine versus cowhage. Further investigation of these mechanisms is crucial to the rational development of strategies to interfere with itch transmission, particularly for the various types of clinical itch that are resistant to antihistamines.

Responses of monkey C-polymodal nociceptors (Johanek et al., 2008) and STT neurons (Davidson et al., 2007) to repeated application of cowhage did not exhibit significant tachyphylaxis. There was also no significant tachyphylaxis to scratching elicited 

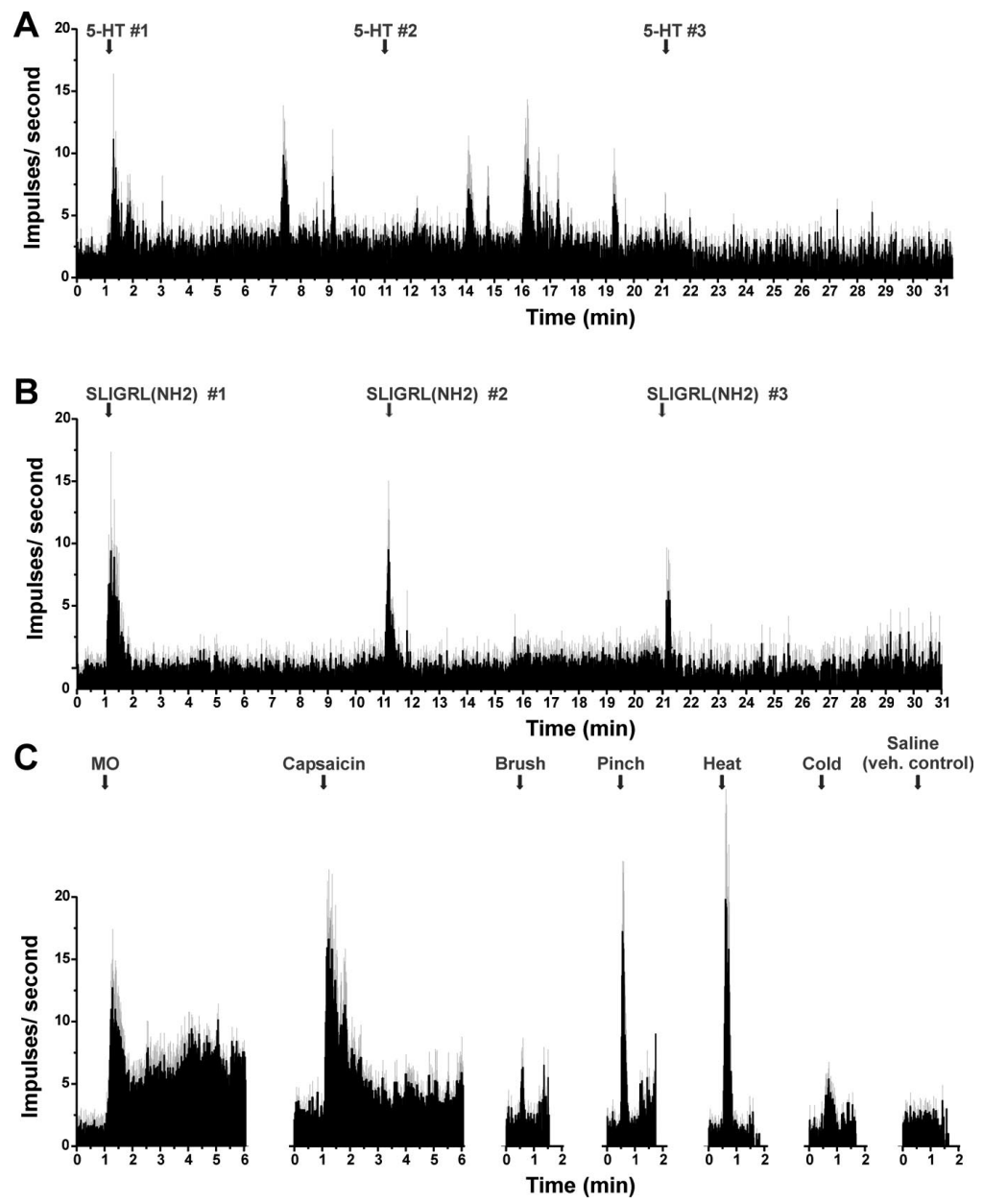

Figure 7. Responses to pruritic and algesic stimuli for superficial dorsal horn units isolated using a 5-HT search strategy (format as in Fig. 4). $\boldsymbol{A}$, Averaged response of seven units to three successive id injections of 5- $\mathrm{HT}$ at 10 min intervals. $\boldsymbol{B}$, Mean responses of units in $\boldsymbol{A}$ to three successive injections of SLIGRL-NH 2 . $\boldsymbol{C}$, Responses of units in $\boldsymbol{A}$ and $\boldsymbol{B}$ to mustard oil, capsaicin, mechanical and thermal stimuli, and id saline [vehicle (veh) control].

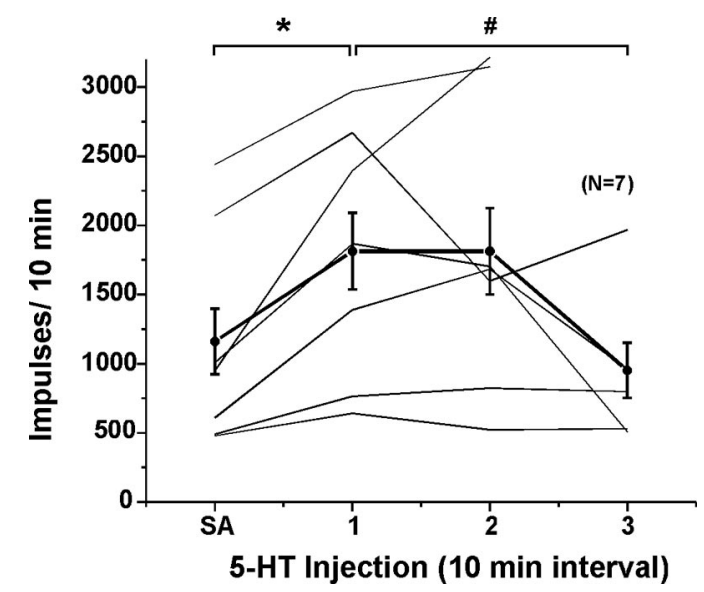

Figure 8. Responses of lumbar superficial dorsal horn units to repeated id microinjections of 5-HT. Format as in Figure 6. ${ }^{*}$ Mean response to first injection of 5-HT significantly different from $\mathrm{SA}(p<0.05, \mathrm{ANOVA})$. "First response to 5-HT significantly different from third ( $p<0.05)$; i.e., tachyphylaxis. Third mean response not significantly different from SA.

by repeated injections of SLIGRL- $\mathrm{NH}_{2}$ at a 40 min interval (Iodi Carstens et al., 2008), in partial contrast to the present data showing a significant $25 \%$ decrease in the mean response of superficial

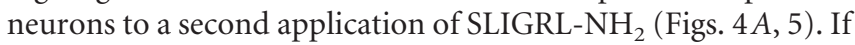

such neurons are involved in itch-related scratching, the $25 \%$ change in mean firing may be insufficient to be expressed behaviorally.

\section{5-HT}

A minority (41\%) of units isolated by the 5-HT search stimulus responded to a second injection of 5-HT. Lack of neuronal response may be attributed to absence of input from 5-HT-sensitive afferents and/or tachyphylaxis. Indeed, neuronal responses exhibited significant tachyphylaxis to repeated 5-HT (Figs. $4 B, 7 A, 8$ ), and we observed significant tachyphylaxis for behavioral scratching to repeated injection of 5-HT (our unpublished observations). Furthermore, the 5-HT search stimulus may have elicited prolonged activity, since baseline firing was greater for units searched with 5-HT compared with SLIGRL- $\mathrm{NH}_{2}$ (compare SA in Figs. 8, 5). Despite these limitations, several units responded to subsequent 5-HT, indicating that tachyphylaxis was incomplete. In addition, there was no significant crosstachyphylaxis between 5-HT and SLIGRL$\mathrm{NH}_{2}$, since a high percentage of neurons isolated by each agent responded to the other one (Table 1, Fig. 7). By the same reasoning, neither 5-HT nor SLIGRL-NH exhibited significant cross-tachyphylaxis to mustard oil or capsaicin.

Identification of itch-signaling neurons A prerequisite for an itch-signaling neuron would be that it responds to the itch mediator over a time course matching that of itch sensation or related scratching behavior. Presently, superficial neurons exhibited significantly increased firing for 9-10 min after id injections of the PAR-2 agonist or 5-HT. However, scratching behavior persisted for 20-30 min after id injection of the PAR-2 agonist (Fig. $2 \mathrm{~B}$ ) or $5-\mathrm{HT}$ (Cuellar et al., 2003). There are several explanations for the briefer duration of neuronal responses versus scratching. (1) Tachyphylaxis of neuronal responses caused by the search stimulus would reduce subsequent responses to the same stimulus. (2) Anesthesia in electrophysiology experiments may have blunted neuronal response duration. (3) Use of a smaller injection volume in electrophysiology versus behavioral experiments. (4) Different injection sites in behavioral (nape of neck) versus electrophysiological (hindpaw) studies. However, hindpaw injection of 5-HT evoked itch-related biting over a 20-30 min period (Hagiwara et al., 1999), similar to that of PAR-2 agonistevoked scratching observed presently. Conceivably, the spinal neuronal response may initiate itch and scratching, which are subsequently maintained by supraspinal mechanisms, even after spinal neuronal activity wanes. Thus, the present data are consistent with the possibility that pruritigen-responsive superficial dorsal horn neurons contribute to itch and the initiation of scratching.

The present search strategy was purposefully biased toward isolating chemosensitive units, allowing the identification of 
superficial dorsal horn neurons responsive to the PAR-2 agonist and 5-HT. This strategy should reveal neurons selectively responsive to the agent, if they exist. However, we found no neurons that responded exclusively to the PAR-2 agonist or 5-HT. Instead, the vast majority of units additionally responded to mustard oil and/or capsaicin, thus exhibiting broad tuning for algesic and pruritic mediators. This is consistent with our previous study of rat superficial dorsal horn units that responded to 5-HT over a time course consistent with behavioral scratching (Jinks and Carstens, 2002). These units additionally responded to algogens mustard oil and capsaicin (Jinks and Carstens, 2002). Similarly, $\sim 50 \%$ of identified primate STT WDR and high-threshold neurons responded to id histamine but nearly all additionally responded to capsaicin (Simone et al., 2004). A more recent study from the same group found that subpopulations of superficial and deep dorsal horn primate STT neurons responded to histamine or cowhage but not both (Davidson et al., 2007a). In the study of cat lamina I STT neurons that responded to cutaneous application of histamine, two of four units tested also responded to mustard oil (Andrew and Craig, 2001). Thus, regardless of whether units are identified using an unbiased antidromic stimulation approach or a heavily biased chemical search strategy, the available data suggest that neurons selectively responsive to one chemical agent are rare and that the majority of spinal neurons responds nonselectively to multiple pruritic and algesic agents. These findings argue against the dominance of an itch-selective pathway and suggest instead that itch may be signaled (at least partly) by chemically nonselective neurons. A population-coding mechanism consistent with the neurophysiologic data is illustrated in Figure 9. In this scenario, itch is signaled by the activation of subpopulations of spinal WDR and NS neurons responsive to both pruritogens and algogens. Noxious stimulation additionally recruits larger populations of pruritogen-insensitive WDR and NS neurons that signal pain while simultaneously masking or occluding itch sensation.

Pruritogen-evoked responses are also expected to be inhibited by scratching of the receptive field but not by administration of the analgesic morphine which can induce or enhance itch (Carstens, 1997). Consistent with this, scratching inhibited the ongoing activity of monkey STT neurons after id histamine and cowhage (Davidson et al., 2007b). Moreover, systemic morphine did not reduce 5-HT-evoked scratching behavior or spinal Fos expression in rats, although it significantly reduced capsaicinevoked spinal Fos expression (Nojima et al., 2003). Systemic morphine $(1 \mathrm{mg} / \mathrm{kg})$ did not affect, whereas the $\mu$-opioid antagonist naltrexone significantly attenuated, SLIGRL-NH $\mathrm{H}_{2}$-evoked scratching behavior in mice (Iodi Carstens et al., 2008). These observations further support a role for PAR-2 agonist- and 5-HTresponsive neurons in the central transmission of itch, and it will be of future interest to determine if such neuronal responses are modulated by opioid agonists and antagonists in a manner consistent with itch.

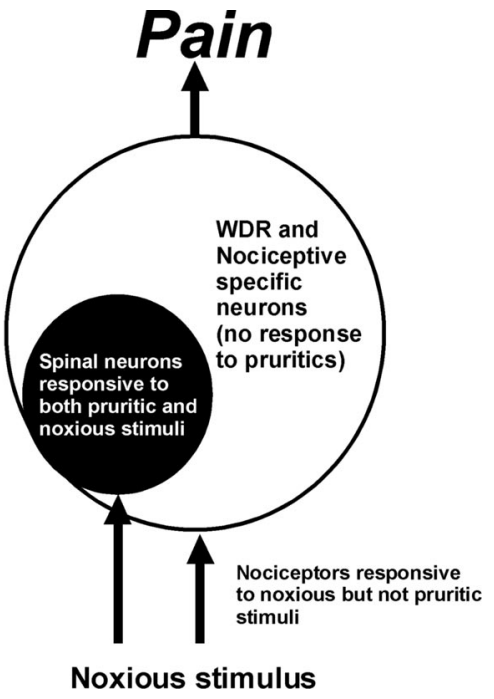

Itch

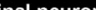

esponsive to

oth pruritic and

oxious stimuli

4

uritic stimulus

Figure 9. Population coding of itch versus pain. Pruritogens excite primary afferent fibers, which in turn excite WDR, NS, and mechano-insensitive neurons in the superficial dorsal horn that transmit itch (left). Noxious stimulation excites nociceptors to

\section{References}

Andrew D, Craig AD (2001) Spinothalamic lamina I neurons selectively sensitive to histamine: a central neural pathway for itch. Nat Neurosci 4:72-77.

Carstens E (1997) Responses of rat spinal dorsal horn neurons to intracutaneous microinjection of histamine, capsaicin, and other irritants. J Neurophysiol 77:2499-2514.

Carstens E, Merrill AW, Akiyama T, Iodi Carstens M (2008) Intradermal PAR-2 agonist excites superficial dorsal horn neurons in the mouse: potential role in itch. Soc Neurosci Abstr 34:771.10/MM1.

Cottrell GS, Amadesi S, Schmidlin F, Bunnett N (2003) Protease-activated receptor 2: activation, signalling and function. Biochem Soc Trans 31:1191-1197.

Cuellar JM, Jinks SL, Simons CT, Carstens E (2003) Deletion of the preprotachykinin A gene in mice does not reduce scratching behavior elicited by intradermal serotonin. Neurosci Lett 339:72-76.

Cuellar JM, Antognini JF, Carstens E (2004) An in vivo method for recording single unit activity in lumbar spinal cord in mice anesthetized with a volatile anesthetic. Brain Res Brain Res Protoc 13:126-134.

Dai Y, Moriyama T, Higashi T, Togashi K, Kobayashi K, Yamanaka H, Tominaga M, Noguchi K (2004) Proteinase-activated receptor 2-mediated potentiation of transient receptor potential vanilloid subfamily 1 activity reveals a mechanism for proteinase-induced inflammatory pain. J Neurosci 24:4293-4299.

Davidson S, Zhang X, Yoon CH, Khasabov SG, Simone DA, Giesler GJ Jr (2007a) The itch-producing agents histamine and cowhage activate separate populations of primate spinothalamic tract neurons. J Neurosci 27:10007-10014.

Davidson S, Zhang X, Khasabov SG, Simone DA, Giesler GJ Jr (2007b) Responses and modulation of monkey spinothalamic tract neurons to itchproducing and itch-inhibiting stimuli. Acta Derm Venereol 87:475.

Hagiwara K, Nojima H, Kuraishi Y (1999) Serotonin-induced biting of the hind paw is itch-related response in mice. Pain Res 14:53-59.

Ikoma A, Steinhoff M, Ständer S, Yosipovitch G, Schmelz M (2006) The neurobiology of itch. Nat Rev Neurosci 7:535-547.

Iodi Carstens M, Akiyama T, Merrill AW, Zanotto KL, Carstens E (2008) Scratching behavior and Fos expression in superficial dorsal horn elicited by protease-activated receptor agonists and other itch mediators in mice. Soc Neurosci Abstr 34:771.11/MM2.

Jinks SL, Carstens E (2000) Superficial dorsal horn neurons identified by intracutaneous histamine: chemonociceptive responses and modulation by morphine. J Neurophysiol 84:616-627.

Jinks SL, Carstens E (2002) Responses of superficial dorsal horn neurons to intradermal serotonin and other irritants: comparison with scratching behavior. J Neurophysiol 87:1280-1289. 
Johanek LM, Meyer RA, Hartke T, Hobelmann JG, Maine DN, LaMotte RH, Ringkamp M (2007) Psychophysical and physiological evidence for parallel afferent pathways mediating the sensation of itch. J Neurosci 27:7490-7497.

Johanek LM, Meyer RA, Friedman RM, Greenquist KW, Shim B, Borzan J, Hartke T, LaMotte RH, Ringkamp M (2008) A role for polymodal C-fiber afferents in nonhistaminergic itch. J Neurosci 28:7659-7669.

Merrill AW, Barter LS, Rudolph U, Eger EI 2nd, Antognini JF, Carstens MI, Carstens E (2006) Propofol's effects on nociceptive behavior and spinal c-fos expression after intraplantar formalin injection in mice with a mutation in the gamma-aminobutyric acid-type(A) receptor beta3 subunit. Anesth Analg 103:478-483.

Nakano T, Andoh T, Lee JB, Kuraishi Y (2008) Different dorsal horn neurons responding to histamine and allergic itch stimuli. Neuroreport 19:723-726.

Namer B, Carr R, Johanek LM, Schmelz M, Handwerker HO, Ringkamp M (2008) Separate peripheral pathways for pruritus in man. J Neurophysiol 100:2062-2069.

Nojima H, Carstens E (2003a) 5-Hydroxytryptamine (5-HT)2 receptor involvement in acute 5 -HT-evoked scratching but not in allergic pruritus induced by dinitrofluorobenzene in rats. J Pharmacol Exp Ther 306:245-252.

Nojima H, Carstens E (2003b) Quantitative assessment of directed hind limb scratching behavior as a rodent itch model. J Neurosci Methods 126:137-143.

Nojima H, Simons CT, Cuellar JM, Carstens MI, Moore JA, Carstens E (2003) Opioid modulation of scratching and spinal c-fos expression evoked by intradermal serotonin. J Neurosci 23:10784-10790.
Reddy VB, Iuga AO, Shimada SG, LaMotte RH, Lerner EA (2008) Cowhageevoked itch is mediated by a novel cysteine protease: a ligand of proteaseactivated receptors. J Neurosci 28:4331-4335.

Schmelz M, Schmidt R, Bickel A, Handwerker HO, Torebjörk HE (1997) Specific C-receptors for itch in human skin. J Neurosci 17:8003-8008.

Shimada SG, Shimada KA, Collins JG (2006) Scratching behavior in mice induced by the proteinase-activated receptor-2 agonist, SLIGRL-NH2. Eur J Pharmacol 530:281-283.

Simone DA, Zhang X, Li J, Zhang JM, Honda CN, LaMotte RH, Giesler GJ Jr (2004) Comparison of responses of primate spinothalamic tract neurons to pruritic and algogenic stimuli. J Neurophysiol 91:213-222.

Steinhoff M, Neisius U, Ikoma A, Fartasch M, Heyer G, Skov PS, Luger TA, Schmelz M (2003) Proteinase-activated receptor-2 mediates itch: a novel pathway for pruritus in human skin. J Neurosci 23:6176-6180.

Thomsen JS, Petersen MB, Benfeldt E, Jensen SB, Serup J (2001) Scratch induction in the rat by intradermal serotonin: a model for pruritus. Acta Derm Venereol 81:250-254.

Twycross R, Greaves MW, Handwerker H, Jones EA, Libretto SE, Szepietowski JC, Zylicz Z (2003) Itch: scratching more than the surface. QJM 96:7-26.

Ui H, Andoh T, Lee JB, Nojima H, Kuraishi Y (2006) Potent pruritogenic action of tryptase mediated by PAR-2 receptor and its involvement in anti-pruritic effect of nafamostat mesilate in mice. Eur J Pharmacol 530:172-178.

Yamaguchi T, Nagasawa T, Satoh M, Kuraishi Y (1999) Itch-associated response induced by intradermal serotonin through 5 -HT2 receptors in mice. Neurosci Res 35:77-83. 